\title{
Antiferromagnetic ground state with pair-checkboard order in FeSe
}

\author{
Hai-Yuan Cao, Shiyou Chen, Hongjun Xiang, Xin-Gao Gong \\ Key Laboratory for Computational Physical Sciences (MOE), \\ State Key Laboratory of Surface Physics, and Department of Physics, \\ Fudan University, Shanghai 200433, China
}

\begin{abstract}
Monolayer FeSe thin film grown on $\mathrm{SrTiO}_{3}(001)$ (STO) shows the sign of $\mathrm{T}_{c}>77 \mathrm{~K}$, which is higher than the $\mathrm{T}_{c}$-record of $56 \mathrm{~K}$ for the bulk FeAs-based superconductors. However, little is known about the magnetic ground state of FeSe, which should be closely related to its unusual superconductivity. Previous studies presume the collinear stripe antiferromagnetic (AFM) state as the ground state of FeSe, same to that in FeAs superconductors. Here we find a novel magnetic order named "pair-checkboard AFM" as the magnetic ground state of tetragonal FeSe. The novel pair-checkboard order results from the interplay between the nearest, the next-nearest and the unnegligible next-next-nearest neighbor magnetic exchange couplings of Fe atoms. The monolayer FeSe in pair-checkbord order shows an unexpected insulating behavior with a Dirac-cone-like band structure related to the specific orbital order of $\mathrm{d}_{x z}$ and $\mathrm{d}_{y z}$ characters of Fe atoms, which could explain recently observed insulatorsuperconductor transition. The present results cast new insights on the magnetic ordering in FeSe monolayer and its derived superconductors.
\end{abstract}

PACS number: 74.25.Ha, 75.70.Ak, 74.70.-b, 73.22.-f 
The high temperature (high- $\mathrm{T}_{c}$ ) superconductivity discovered in the iron-based superconductors [1,3] breaks the conventional knowledge that the magnetic atoms like Fe should not contribute to the superconductivity. This inspires that the magnetism plays an important role in the mechanism of the high- $\mathrm{T}_{c}$ superconductivity in iron-based superconductors [4]. Although the electronic properties for different families of iron-based superconductors can be somehow different [5], they all are believed to share the common feature of AFM ordered parent compound [6].

While the magnetism contributing to the high-Tc superconductivity has attracted wide attention [7], the magnetic ground states for the parent compounds of iron-based superconductors remain unclear. Recently, the sign of over $77 \mathrm{~K}$ unconventional high $\mathrm{T}_{c}$ superconductivity [8-11] has been observed in monolayer FeSe grown on STO substrate [12 17], which is much higher than the highest $\mathrm{T}_{c}$ record in the intensively studied FeAs systems [18, 19]. For FeAs-based materials, the collinear AFM (or the stripe AFM) has been verified as the ground state for the parent compounds by neutron scattering [7]. However, the ground state for the compound based on FeSe is still waiting to be clarified. Previous theoretical studies presumed that FeSe has the same ground state as FeAs-based materials [20 24]. From the previous experimental results, the electronic properties of FeSe-based materials are much different from that of FeAs-based materials [25], especially for that in monolayer FeSe on STO substrate [8 17]. Recent ARPES experiment observed that the insulatorsuperconductor transition via doping in the monolayer FeSe grown on the STO substrate [17], which indicates that the ground state of monolayer FeSe could be insulating. Besides, in the recently discovered molecular-intercalated iron-selenide $\mathrm{Li}_{x}\left(\mathrm{ND}_{2}\right)_{y}\left(\mathrm{ND}_{3}\right)_{1-y} \mathrm{Fe}_{2} \mathrm{Se}_{2}$ [26], the neutron-inelastic-scattering measurements found that the magnetic scattering in momentum space is unusually closer to wave-vector $\left(\pi, \frac{\pi}{2}\right)$ [27], which means that in FeSe there could be an unexpected magnetic order other than collinear AFM. As yet, unveiling the ground-state properties of FeSe would be crucial for understanding the mechanism of novel high-Tc superconductivity.

Here, based on the first-principles calculations, we find a novel magnetic order, named as the pair-checkboard AFM, which is quite different from previously proposed magnetic order in iron based compounds, to be the magnetic ground state (Fig. 1(a)) for tetragonal FeSe [28]. The underlying physics of this new magnetic order could be effectively described by a Heisenberg model including the nearest, the next-nearest and the next-next-nearest 
neighbor superexchange interaction mediated by Se-4p orbitals. This novel ground state is metallic in the bulk FeSe, while it has a Dirac-cone-like band structure with a non-zero band gap induced by the spin-orbit interaction in monolayer FeSe, where the interlayer interaction between the FeSe layers is absent. The pair-checkboard AFM would also induce a $2 \times 1$ reconstruction in FeSe due to the different distance of the Se atoms to the Fe plane. Furthermore, we confirm that this novel pair-checkboard AFM not only exists in tetragonal FeSe but also in its derived compounds, like the recently synthesized bulk $\mathrm{LiFeO}_{2} \mathrm{Fe}_{2} \mathrm{Se}_{2}$ [29]. This is the first time to reveal that the magnetic ground state of FeSe is different from that of FeAs-based materials, which could well explain several intriguing experimental observations.

In this letter, we performed extensive study on the electronic and magnetic properties of FeSe based on the first-principles simulations. We employed the plane-wave basis set and the projected augmented wave method [30, 31] which is implemented in the VASP code [32, 33. to calculate the electronic and magnetic properties. We adopted the generalized gradient approximation (GGA) with Perdew-Burke-Ernzerhof (PBE) formula [34] for the exchangecorrelation functional. A plane-wave cutoff energy of $450 \mathrm{eV}$ and a Monkhorst-Pack mesh of $16 \times 8 k$-points [35] for monolayer FeSe and $18 \times 9 \times 9 k$-points for bulk FeSe with 0.1 $\mathrm{eV}$ Gaussian smearing were used in magnetic unit cell calculations. A supercell of $16 \mathrm{Fe}$ atoms was used to calculate the magnetic exchange coupling parameters. The vacuum layer more than $20 \AA$ thick was used in the calculation for monolayer FeSe to ensure decoupling between neighboring FeSe layers. For structural relaxation, all the atoms were allowed to relax until atomic forces are smaller than $0.01 \mathrm{eV} / \AA$. The density of states calculations were performed based on the tetrahedral method [36] with a much denser k-grid of $24 \times 12$ and $24 \times 12 \times 12$ for the magnetic unit cell of monolayer FeSe and bulk FeSe, respectively. Both the lattice constant and the atomic positions are fully optimized.

Our results show that the magnetic ground state of FeSe should be the pair-checkboard AFM, rather than the collinear AFM which was assumed to be. We have calculated the relative energy of various magnetic orders, including the nonmagnetic state, the checkboard AFM order (or the Nïœeel AFM), the bicollinear AFM order, the collinear AFM order and the pair-checkboard AFM. Table I lists the energies and the magnetic moments for both the bulk FeSe and monolayer FeSe. One can clearly see that the energy of pair-checkboard AFM is lower than that of the collinear AFM order by $15 \mathrm{meV} /$ f.u. and $12 \mathrm{meV} /$ f.u. for the bulk 
FeSe and monolayer FeSe, respectively, while the magnetic moments in the bulk FeSe and monolayer FeSe are quite similar. The breaking of the $\mathrm{C}_{4}$ symmetry after lattice-constant optimization is found in the pair-checkboard AFM order as well as in the collinear AFM, which is caused by the ferro-orbital order of Fe atoms in FeSe [37].

The pair-checkboard AFM is different from the collinear AFM order that each spin of Fe atom has one neighbor spin aligned ferromagnetically while the other three neighbor spins all aligned antiferromagnetically (Fig. 1a). More interestingly, we find apparent difference between the charge-density distribution of the pair-checkboard AFM and the collinear AFM (Fig. 1b, 1c). Both Fe and Se atoms in the pair-checkboard AFM order has a unique orbital order which does not appear in other AFM orders. The Se atoms could be divided into two groups (labeled as Se1 and Se2 in Fig. 1(b)) with the different distances between the Se atoms and the Fe plane $\left(z_{S e}\right)$. The $z_{S e}$ for Se1 is $1.48 \AA$ and that for Se2 is $1.46 \AA$ which can be regarded as a $2 \times 1$ reconstruction in FeSe.

The electronic band structure and the projected PDOS of bulk and monolayer FeSe with the pair-checkboard AFM state is shown in Fig. 2 and Fig. 3, respectively. For bulk FeSe, the band structure shows that there are two electron pokets locating around $\Gamma$ point and along $X^{\prime}-\Gamma$ line, and one hole pocket centered around $Z$ point. From the projected PDOS, we find the band crossing the Fermi level mainly coming from the $d_{x z}$ and $d_{y z}$ orbitals, which is caused by the interlayer interaction between adjacent FeSe layers in bulk FeSe. The PDOS of Fe atoms near the Fermi level looks similar for both bulk FeSe and monolayer FeSe, which means that the intralayer interaction in FeSe layers could probably dominate the major physics related to the superconductivity in FeSe.

The tetragonal FeSe with pair-checkboard AFM order has a feature of exotic Dirac-conelike band structure as shown in Fig. 2(a) and 2(b), respectively. If including the spin-orbit coupling, monolayer FeSe with the pair-checkboard AFM becomes insulating with a band gap around $27 \mathrm{meV}$, while that with other magnetic orders are all metallic. It is worth to note that both the valence band maximum (VBM) and the conduction band minimum $(\mathrm{CBM})$ of the band structure locating around the k-point $(0.2,0.0,0.0)$ in $\Gamma-X$ boundary of the first magnetic Brillouin zone.

The Dirac-cone-like band structure relates to a specific orbital order of $d_{x z}$ and $d_{y z}$ characters of Fe atoms, which can be seen from the decomposed band structure near the Fermi level (see Supplemental Material Figure S2 [38]). The spin-majority $d$-orbitals of Fe atoms 
are almost all filled, while the density of states (DOS) near the Fermi level is mostly contributed by the spin-minority $d$-orbitals of the Fe atoms. For the spin-minority part of Fe atoms, $d_{x^{2}-y^{2}}, d_{z^{2}}$ and $d_{x z}$ orbitals are mostly filled while $d_{y z}$ and $d_{x y}$ orbitals are slightly filled (Fig. 3(b)). The two bands cross over the Fermi level are mainly composed of spinminority $d_{x z}$ and $d_{y z}$ characters of Fe atoms, respectively. If without the spin-orbit coupling, $d_{x z}$ and $d_{y z}$ belong to different symmetry-groups which allows them to cross without hybridization at the Fermi level. Then turning on the spin-orbit coupling could lead to the mixing between $d_{x z}$ and $d_{y z}$ orbitals, resulting a gap opening. The charge density at the VBM is mainly composed of $d_{x z}$ hybridized with $d_{x y}$ and $d_{z^{2}}$ of Fe atoms, while that at the CBM is mainly composed of $d_{y z}$ hybridized with $d_{x y}$ (Fig. 3(b)). The charge-density distribution at the VBM and CBM is in agreement with the ferro-orbital order of $d_{x z}$ and $d_{y z}$ orbitals[37. The emerging Dirac-cone-like band structure in the pair-checkboard AFM should be directly related to the novel magnetic ground state in the monolayer FeSe, which means that the orbital order and the magnetic order are strongly coupled together.

To further describe the origin of the new magnetic order in FeSe quatitatively, we propose a frustrated Heisenberg model with the nearest, the next-nearest and the next-next-nearest neighbor couplings $J_{1}, J_{2}$ and $J_{3}[23$.

$$
H=J_{1} \sum_{<i j\rangle} \vec{S}_{i} \cdot \vec{S}_{j}+J_{2} \sum_{<<i j>>} \vec{S}_{i} \cdot \vec{S}_{j}+J_{3} \sum_{<<<i j>>>} \vec{S}_{i} \cdot \vec{S}_{j}
$$

whereas $\langle i j\rangle,\langle<i j\rangle>$ and $\langle<\langle i j\rangle>>$ denote the summation over the nearest, the next-nearest and the next-next-nearest neighbors, respectively. The present model includes the next-next-nearest magnetic coupling $J_{3}$, and this is crucial to correctly describe the new magnetic ground state in FeSe. According to the band structure of FeSe, we believe that our Heisenberg model could capture the substantial physics in the magnetic properties. Mapping from the calculated energy of different mangetic orders, we find that for bulk FeSe $J_{1}=47 \mathrm{meV} / \mathrm{S}^{2}, J_{2}=27 \mathrm{meV} / \mathrm{S}^{2}$, and $J_{3}=7 \mathrm{meV} / \mathrm{S}^{2}$, while for monolayer FeSe $J_{1}=44$ $\mathrm{meV} / \mathrm{S}^{2}, J_{2}=25 \mathrm{meV} / \mathrm{S}^{2}$, and $J_{3}=6 \mathrm{meV} / \mathrm{S}^{2}$.

It is known that when $J_{2}>\frac{J_{1}}{2}$, the collinear AFM rather than the checkboard AFM would be the magnetic ground state in a $J_{1}-J_{2} \mathrm{AFM}$ square lattice. If further including $J_{3}>\frac{J_{2}}{2}$ in the model, the magnetic ground state would turn out to be the bicollinear AFM 23]. In our calculation for FeSe, we find that $J_{2}>\frac{J_{1}}{2}$ and $\frac{J_{2}}{2}>J_{3}>\frac{2 J_{2}-J_{1}}{2}$, and under 
this condition the new magnetic order we proposed turns out to be the magnetic ground state in FeSe.

$\mathrm{LiFeO}_{2} \mathrm{Fe}_{2} \mathrm{Se}_{2}$, a FeSe-based superconductor with $\mathrm{T}_{c} \sim 43 \mathrm{~K}$ and neutral $\mathrm{LiFeO}_{2}$ antiPbO-type spacer layers intercalated between FeSe layers, was recently synthesized by the hydrothermal method [29]. The relative energy difference between various magnetic orders in $\mathrm{LiFeO}_{2} \mathrm{Fe}_{2} \mathrm{Se}_{2}$ is similar to that in FeSe (see Supplemental Material Table S1 [38]). The calculation shows that the pair-checkboard AFM is not only the magnetic ground state for $\mathrm{LiFeO}_{2} \mathrm{Fe}_{2} \mathrm{Se}_{2}$, but its relative stability to other magnetic states is even more robust comparing to that of monolayer FeSe. This reveals that the pair-checkboard AFM could be the universal magnetic ground state for FeSe layer and its derived undoped materials. Besides that, we also find that the Dirac-cone-like band structure still be kept in the bulk $\mathrm{LiFeO}_{2} \mathrm{Fe}_{2} \mathrm{Se}_{2}$.

In previous experiments, there was no direct observation for the pair-checkboard AFM order in bulk tetragonal FeSe. This could probably be attributed to the absense of the high quality FeSe sample. The extra Fe atoms in the FeSe samples could suppress the stability of the pair-checkboard AFM order (see Supplemental Material Figure S4[38]). Although the pair-checkboard AFM order has not been observed directly yet, there were several experimental evidences indicating the existence of this novel magnetic order. At first, the $27 \mathrm{meV}$ band gap we predicted in FeSe monolayer with pair-checkboard AFM order is quite closed to the ARPES experimentally observed insulating gap of $20^{\sim} 25 \mathrm{meV}$ [17], while that with other magnetic orders all remains to be metallic. Another STM experiment observed a $2 \times 1$ reconstruction would occur in monolayer FeSe on the STO substrate [39], which is also coincident with two types of Se atoms with different height $z_{S e}$ in pair-checkboard AFM. Very recently, electric transport measurements observed the Dirac-cone-like ultrafast carriers in the single crystal FeSe superconductor [40], which could also be interpreted as originating from our proposed pair-checkboard AFM order induced Dirac-cone-like band structure.

In summary, the present studies reveal that FeSe does not share the same collinear AFM magnetic ground state with the FeAs-based materials. The magnetic ground state of FeSe is pair-checkboard AFM, which is metallic in bulk FeSe and insulating with a 27 meV band gap in monolayer FeSe and completely different from the magnetic states found in other iron-based superconductors. Such novel magnetic order is found to be robust against tensile strain up to a few percent and also robust against electron doping to a certain level (see 
Supplemental Material Figure S4 [38]). The properties of the predicted gapped insulating ground state are in good agreement with the recent experimental observations [17, 39, 40]. The pair-checkboard AFM order in FeSe shed new lights on the understanding of high$\mathrm{T}_{c}$ superconductivity in FeSe monolayer on the oxides substrates and FeSe-layer derived superconductors. The novel pair-checkboard AFM order we predicted would call more direct experiments for investigating the magnetic properties in high-quality FeSe samples.

\section{Acknowledgments}

We acknowledge professor D. L. Feng, Dr. Rui Peng and Dr. Shiyong Tan for stimulating discussions. The work was partially supported by the Special Funds for Major State Basic Research, National Natural Science Foundation of China (NSFC), Program for Professor of Special Appointment (Eastern Scholar) and the National Basic Research Program of China (973 Program). Computation was performed in the Supercomputer Center of Fudan University. 


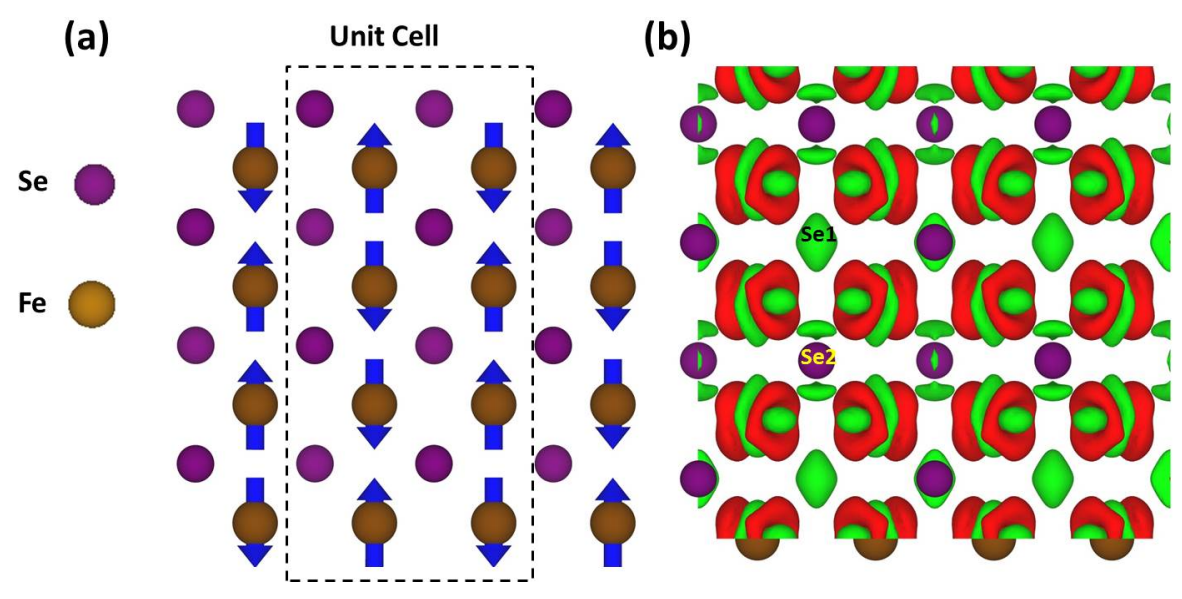

(c)

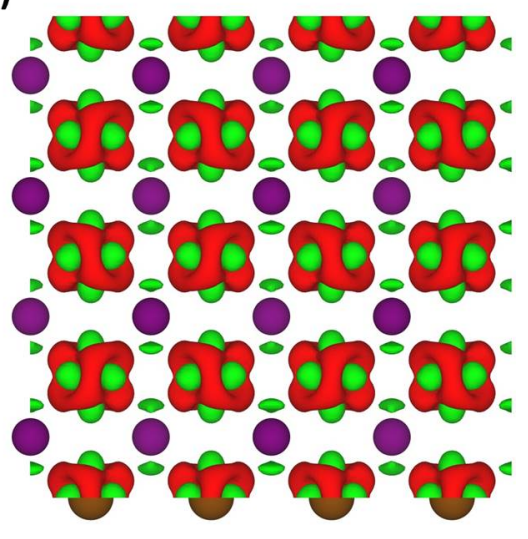

Figure 1: (a) Schematic top view of the pair-checkboard AFM in FeSe layer. Each spin of Fe atom has only one neighbor spin aligned ferromagnetically while the other neighbor spins all aligned antiferromagnetically. The rectangle enclosed by the dashed lines denotes the magnetic unit cell. (b) The charge density difference between the pair-checkboard AFM and the nonmagnetic state. (c) The charge density difference between the collinear AFM and the nonmagnetic state. The isosurface depicted by the red and green colors represents the lost and gained charge density comparing to the nonmagnetic state. Se atoms in the pair-checkboard AFM order show an orbital order which are labeled with Se1 (blue) and Se2 (yellow), respectively. 


\begin{tabular}{|c|c|c|c|c|}
\hline & $\Delta E_{\text {Bulk }}(\mathrm{meV} /$ f.u. $)$ & $\mathrm{M}_{\text {Bulk }}\left(\mu_{B}\right)$ & $\Delta E_{\text {Mono }}(\mathrm{meV} /$ f.u. $)$ & $\mathrm{M}_{\text {Mono }}\left(\mu_{B}\right)$ \\
\hline \hline Nomangetic Order & 0 & 0 & 0 & 0 \\
\hline Collinear AFM & -69 & 1.9 & -87 & 1.9 \\
\hline Checkboard AFM & -41 & 1.7 & -62 & 1.7 \\
\hline Bicollinear AFM & -21 & 2.2 & -37 & 2.2 \\
\hline pair-checkboard AFM & -84 & 2.0 & -99 & 2.0 \\
\hline
\end{tabular}

Table I: Energy difference $\Delta E$ (reference to the energy of the nonmagnetic state) and magnetic moments $M_{F e}$ of the bulk and monolayer FeSe with different magnetic order. 

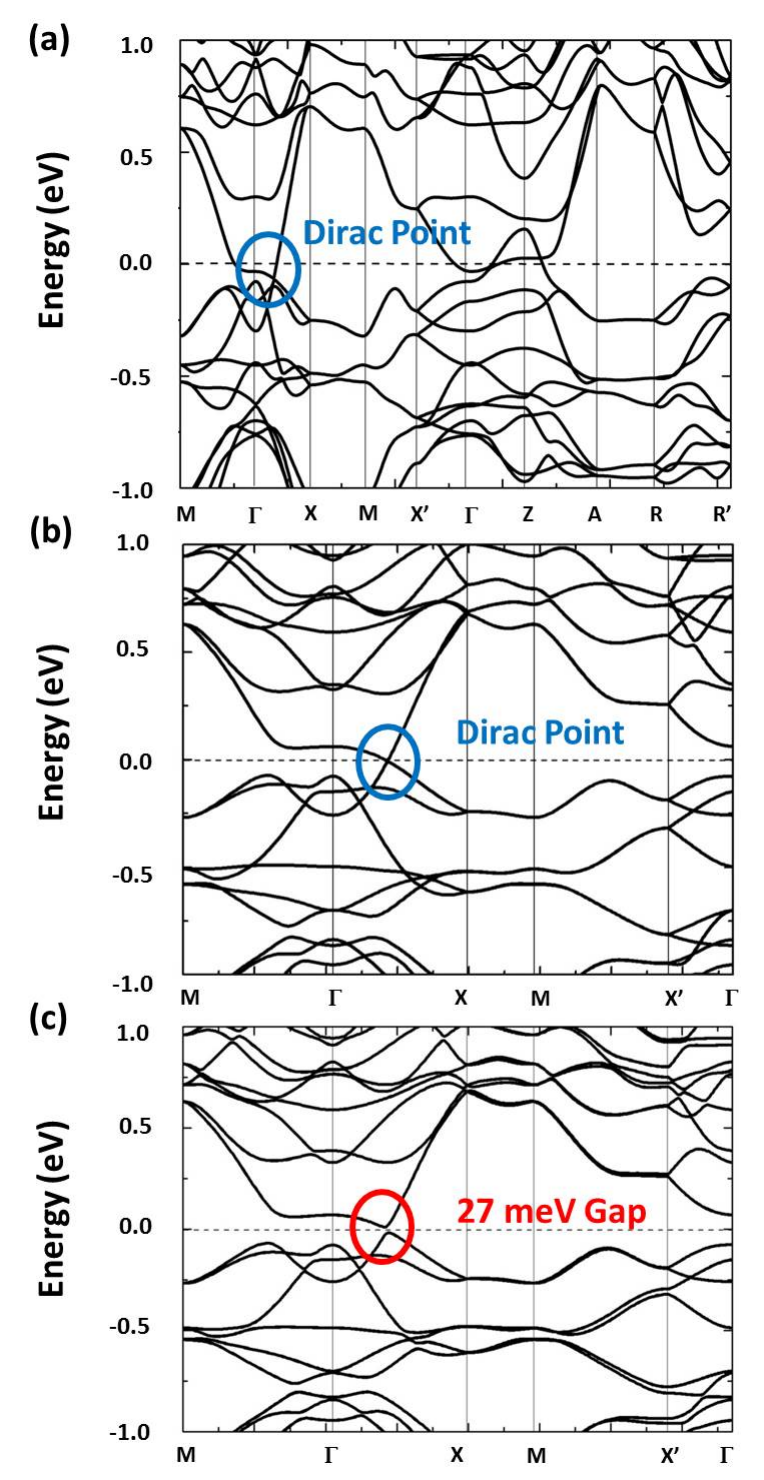

Figure 2: (a) Electronic band structure of bulk FeSe with the pair-checkboard AFM. (b) and (c) Electronic band structure of monolayer FeSe in the pair-checkboard AFM order without or with the spin-orbit coupling. The Dirac-point appears in the bulk FeSe and monolayer FeSe (highlighted), while including SOC would open (highlighted) a $27 \mathrm{meV}$ indirect band gap in monolayer FeSe. The Fermi level is denoted by the dashed line. 

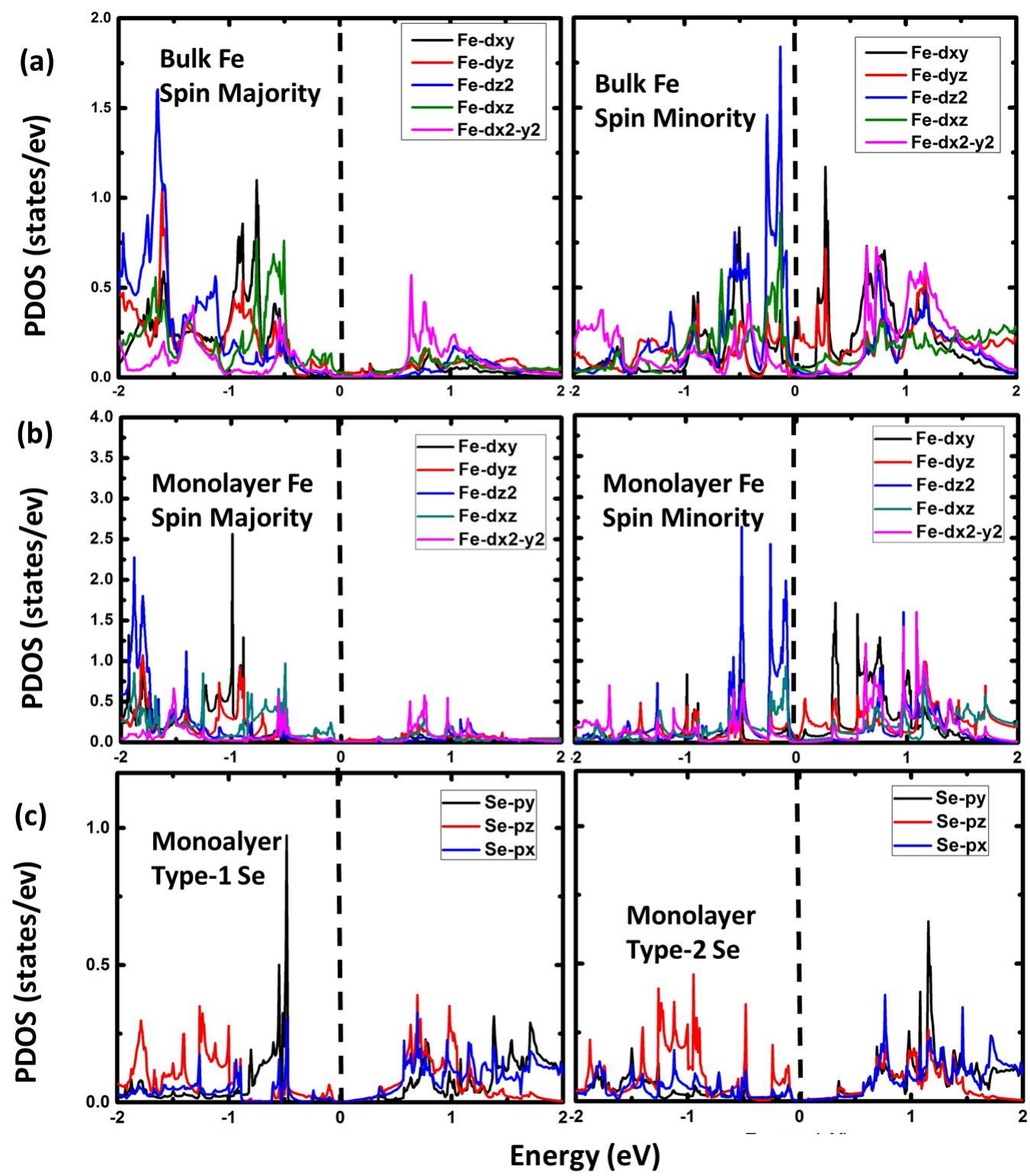

Figure 3: Projected orbital-resolved partial density of states of (a) spin-majority part and spinminority part of Fe atom in bulk FeSe, (b) spin-majority part and spin-minority part of Fe atom in monolayer FeSe, (c) type-1 Se atom and type-2 Se atom (defined in Fig. 3(b)), respectively. The d-orbitals of Fe atoms diminish to 0 at the Fermi level in monolayer FeSe, while the spin-minority part of $d_{x z}$ and $d_{y z}$ orbitals cross the Fermi level in bulk FeSe. The position of the Fermi level is denoted by the dashed lines. 
[1] Y. Kamihara et al., J. Am. Chem. Soc, 130, 3296 (2008).

[2] M. Rotter et al., Phys. Rev. Lett. 101, 107006 (2008).

[3] F.-C. Hsu. et al., Proc. Natl. Acad. Sci. U.S.A 105, 14262 (2008).

[4] D. J. Scalapino, Rev. Mod. Phys. 84, 1383 (2012).

[5] D. C. Johnston, Adv. Phys. 59, 803 (2010).

[6] Y. J. Uemura, Nat. Mater. 8, 253 (2009).

[7] P. Dai et al., Nat. Phys. 8, 709 (2012).

[8] Q. Y. Wang et al., Chin. Phys. Lett. 29, 037402 (2013).

[9] L. Z. Deng et al., arXiv:1311.6459.

[10] R. Peng et al., Nat. Commun. 5, 5044 (2014).

[11] J.-F. Ge et al., arXiv:1406.3435.

[12] D. F. Liu et al., Nat. Commun. 3, 931 (2012).

[13] S. L. He et al., Nat. Mater. 12, 605 (2013).

[14] S. Y. Tan et al., Nat. Mater. 12, 634 (2013).

[15] H. W. Zhang et al., Chin. Phys. Lett. 31, 1(2014).

[16] Xu Liu et al., Nat. Commun. 5, 5047 (2014).

[17] Junfeng He et al., arXiv:1401.7115.

[18] Z. A. Ren et al., Chin. Phys. Lett. 25, 2215 (2008).

[19] C. Wang et al., Europhys. Lett. 83, 67006 (2008).

[20] A. Subedi et al., Phys. Rev. B 78, 134514 (2008).

[21] T. Bazhirov and M. L. Cohen, J. Phys.: Condens. Matter 25, 105506 (2013).

[22] K. Liu, Z.-Y Lu, and T. Xiang, Phys. Rev. B 85, 235123 (2012).

[23] F. Ma et al., Phys. Rev. Lett 102, 177003 (2009).

[24] Hai-Yuan Cao et al., Phys. Rev. B 89, 014501 (2014).

[25] E. Dagotto, Rev. Mod. Phys. 85, 849 (2013).

[26] M. Burrard-Lucas et al., Nat. Mater. 12, 15 (2013).

[27] A. E. Taylor et al., Phys. Rev. B 87, 220508(R) (2013).

[28] D. P. Landau and K. Binder, Phys. Rev. B 31, 5946 (1985).

[29] X. F. Lu et al., Phys. Rev. B 89, 020507(R) (2013). 
[30] P. E. Blochl, Phys. Rev. B 50, 17953 (1994).

[31] G. Kresse and D. Joubert, Phys. Rev. B 59, 1758 (1999).

[32] G. Kresse and J. Furthmï¿œller, Comput. Mat. Sci. 6, 15 (1996).

[33] G. Kresse and J. Furthmï¡œller, Phys. Rev. B 54, 11169 (1996).

[34] J. P. Perdew, K. Burke, and M. Ernzerhof, Phys. Rev. Lett. 77, 3865, (1996).

[35] H. J. Monkhorst and J. D. Pack, Phys. Rev. B 13, 5188 (1976).

[36] Peter E. Blï¿œchl, O. Jepsen, and O. K. Andersen, Phys. Rev. B 49, 16223 (1994).

[37] Chi-Cheng Lee, Wei-Guo Yin, and Wei Ku, Phys. Rev. Lett. 103, 267001 (2009).

[38] See Supplemental Material for the Brillouin zone used in the calculation of band structure, the orbital decomposed band structure, the charge-density plot at the Dirac point for monolayer FeSe, the relative energy of bulk $\mathrm{LiFeO}_{2} \mathrm{Fe}_{2} \mathrm{Se}_{2}$ with different magnetic order and the stability of pair-checkboard AFM order against strain and charge-doping.

[39] Junhyeok Bang et al., Phys. Rev. B 87, 220503(R) (2014).

[40] K. K. Huynh et al., arXiv:1405.3815.

[41] A. E. Bohmer et al., Phys. Rev. B 87, 180505(R) (2013). 